\title{
SEGURANÇA NO TRABALHO DE PREPARO DE CALDA NO TANQUE DE 2.000L DO TURBOPULVERIZADOR COM FORMULAÇÕES LÍQUIDAS DE AGROTÓXICOS REGISTRADAS PARA A CULTURA DE GOIABA ${ }^{1}$
}

\author{
MATHEUS BELLINI TÁCIO², MAURÍCIO LEITE DE OLIVEIRA³, \\ JOAQUIM GONÇALVES MACHADO NETO ${ }^{4}$
}

RESUMO - Na atividade de preparo de caldas no tanque do turbopulverizador, há risco de intoxicação do trabalhador devido à exposição, à toxicidade e à concentração do ingrediente ativo tóxico na formulação. Objetivou-se quantificar as exposições dérmicas e respiratórias diárias às dez formulações líquidas de agrotóxicos registradas para a cultura da goiaba, na atividade de abastecimento de dez tanques de $2.000 \mathrm{~L}$ do turbopulverizador, e a distribuição da exposição dérmica nas partes do corpo do trabalhador, avaliar a eficiência de dois conjuntos de vestimentas hidrorrepelentes, classificar as três condições de trabalho em seguras ou inseguras, e determinar as necessidades de controle das exposições e o número de preparos de caldas seguros. A exposição dérmica na atividade de preparo de caldas é de $13,35 \mathrm{~mL}$ de formulação/dia, e a respiratória, de $0,000153 \mathrm{~mL}$. Nesta atividade de preparo de caldas, as mãos do trabalhador são as partes do corpo mais expostas, com 70,6\% da exposição dérmica. A eficiência do conjunto Agro Light, da empresa R\&B, é de $92,8 \%$ da exposição dérmica diária do preparador de caldas, e do Kit Tratorizado, da Azeredo, 94,2 \%. As exposições dérmicas não controladas pelos conjuntos de proteção individual distribuem-se nas diversas partes do corpo do trabalhador. As atividades de preparo de caldas com as dez formulações líquidas de agrotóxicos registrados na cultura de goiaba são inseguras para o trabalhador. Com as vestimentas de proteção individual, apenas a atividade de preparo de caldas com o inseticida PROVADO (200 g de imidacloprido/L) é segura. Para as condições de trabalho inseguras, as necessidades de controle das exposições variam de 73,8 a $100 \%$ (sem proteção), de 26,9 a 99,7\% (Agro Light) e de 9,4 a 99,6\% (Kit tratorizado). O número de preparo de caldas seguro é de 0 tanques/dia sem proteção, de 0 a 5 ou 7 (Agro Light) ou a 7, 6 ou 9 (Kit Tratorizado). Termos para indexação: agrotóxicos, exposição dérmica, exposição respiratória, goiaba.

\section{WORK SAFETY DURING THE MIXING OF PESTICIDE OF THE 2.000L AIR-ASSISTED SPRAYER TANK WIHT PESTICIDE LIQUID FORMULATIONS REGISTERED IN GUAVA ORCHARDS}

\begin{abstract}
Pure formulations are manipulated during the mixing of pesticide sprayer, involving elevated exposure of workers. The aim was to quantify the dermal and respiratory exposures at ten day liquid formulations of pesticides registered for the guava orchards in the activity of mixing the spray and loading to fill ten tank of 2,000 L of air-assisted sprayer, and distribution of dermal exposure in body parts of the worker; evaluate the efficiency of two sets of personal protective clothing water-repellent to control dermal exposure, and classify the activities with ten formulations in safe or unsafe, with and without the use of personal protective clothing, and determine the exposure control need and the number of mixing of pesticides safe. Dermal exposure in the activity of mixing the spray is $13.35 \mathrm{~mL}$ formulation/day and respiratory, $0,000,153 \mathrm{ml}$. In this activity of mixing the spray, the worker's hands are the body parts most at exposure, with $70.6 \%$ of dermal exposure. The efficiency of the set Agro Light is $92.8 \%$ of the dermal exposure daily and Kit Tratorizado, $94.2 \%$. The dermal exposure not-controlled by sets of personal protective equipment are distributed in different parts of the body of the worker. The activities of mixing the spray with ten liquid formulations of pesticides registered in the guava orchard are unsafe for the worker. With the use of sets of personal protective clothing, only the activity of mixing the spray with PROVADO insecticide (imidacloprid $200 \mathrm{~g} / \mathrm{L}$ ) is safe. For unsafe working conditions, the exposure control need ranging from 73.8 to $100 \%$ (no protection), 26.9 to $99.7 \%$ (Agro Light) and 9.4 to $99.6 \%$ (Kit Tratorizado). The number of mixing of pesticides safe is 0 tanks / day without protection, from 0 to 5 or 7 (Agro Light) or to 6, 7 or 9 (Kit Tratorizado).
\end{abstract}

Index terms: pesticide, dermal exposure, respiratory exposure, guava.

\footnotetext{
1(Trabalho 170-09). Recebido em: 20-07-2009. Aceito para publicação em: 22-02-2010.Trabalho financiado com Auxílio à Pesquisa e Bolsa de Iniciação Científica para o primeiro autor, concedidos FAPESP.

${ }^{2}$ Engenheiro Agrônomo- agrobuck@yahoo.com.br.

${ }^{3}$ Doutor em Agronomia e Engenheiro de Segurança do Trabalho. oliveiraml@hotmail.com

${ }^{4}$ Prof. Dr. da FCAV/UNESP Câmpus de Jaboticabal - joaquim@fcav.unesp.br.
} 


\section{INTRODUÇÃO}

$\mathrm{Na}$ atividade de preparo de calda de agrotóxicos, o trabalhador manuseia a embalagem, quebra o lacre da tampa, retira a tampa, quebra o lacre do bocal da embalagem, dosa a formulação e recoloca a tampa. Na última dosagem, quando a embalagem fica completamente vazia, faz a tríplice lavagem. A formulação dosada normalmente é diluída em água, ou pré-diluída, ou depositada diretamente no tanque do pulverizador.

O risco de intoxicação do trabalhador geralmente é maior nas atividades de manuseio de formulações de agrotóxicos que, nas de aplicação, devido à diluição das formulações em água (VAN HEMMEN, 1992 e OLIVEIRA, 2000). A intensidade do risco de intoxicação dos trabalhadores expostos aos agrotóxicos depende da toxicidade do ingrediente ativo e da exposição proporcionada pelas condições específicas de trabalho. Em condições de campo, $99 \%$, ou mais, da exposição do trabalhador aos agrotóxicos ocorrem na via dérmica e $1 \%$, ou menos, na via respiratória, tanto na agricultura brasileira (OLIVEIRA, 2000; OLIVEIRA; MACHADO NETO, 2003a) como na do exterior (VAN HEMMEN, 1992).

As condições de trabalho com os agrotóxicos são classificadas em seguras ou inseguras, de acordo com o cálculo da margem de segurança (MS) proposto por Severn (1984). As condições de trabalho são classificadas em seguras, se $\mathrm{MS} \geq 1$, ou inseguras, se $\mathrm{MS}<1$. Para as inseguras, Machado-Neto (2001) propôs o calculo da necessidade de controle das exposições para torná-las seguras e do tempo de trabalho seguro, nas atividades de aplicação, ou o número seguro de preparo de caldas ou de abastecimentos de tanques. A partir destes cálculos, podem-se selecionar e recomendar as medidas de segurança mais eficientes, econômicas e confortáveis para o trabalhador.

Em cultura de goiaba no País, apenas um estudo foi realizado na atividade de tratorista $\mathrm{em}$ aplicação de agrotóxicos com turbopulverizador (TÁCIO et al., 2008). Neste trabalho, a exposição dérmica potencial proporcionada ao tratorista foi de $3.807,3 \mathrm{~mL}$ de calda/dia, e a respiratória, de apenas $0,024 \mathrm{~mL}$ de calda/dia. $\mathrm{O}$ conjunto de proteção individual hidrorrepelente Agro Light, da empresa R\&B Equipamentos de Segurança, e o Kit Tratorizado, da AzeredoEPI, controlaram a exposição proporcionada ao tratorista. As vestimentas de proteção individual tornaram seguras as pulverizações de sete $(43,75 \%)$ dos dezessete agrotóxicos considerados. Porém, a segurança da atividade de preparo de caldas no tanque do turbopulverizador para a aplicação de agrotóxicos na cultura da goiaba e a eficiência de vestimentas de proteção individual nesta atividade ainda não foram avaliadas. $\mathrm{Na}$ atividade de preparo de calda, as mãos são as partes do corpo do trabalhador que ficam mais expostas devido ao manuseio da embalagem e da dosagem manual da formulação (VAN HEMMEN, 1992; OLIVEIRA, 2000). De maneira geral, a eficiência de luvas e botas de borracha e de máscara descartável com filtro de carvão ativado é de $95 \%$ (Lundehn et al., 1992).

Objetivou-se quantificar as exposições dérmica e respiratória diárias às dez formulações líquidas de agrotóxicos registradas para a cultura da goiaba, na atividade de abastecimento de dez tanques de 2.000 L do turbopulverizador, e a distribuição da exposição dérmica nas partes do corpo do trabalhador, avaliar a eficiência de dois conjuntos de vestimentas hidrorrepelentes, classificar as três condições de trabalho avaliadas em segura ou insegura, e determinar as necessidades de controle das exposições e o número de preparo de caldas seguros.

\section{MATERIAL E MÉTODOS}

As avaliações das exposições respiratórias e dérmicas do trabalhador, na atividade de preparo de caldas no tanque do turbopulverizador, foram realizadas em uma cultura de goiaba no município de Vista Alegre do Alto - SP. As análises nas vestimentas e materiais de amostragem das exposições foram realizadas no Laboratório de Ecotoxicologia dos Agrotóxicos e Saúde Ocupacional, da Faculdade de Ciências Agrárias e Veterinárias - UNESP - Câmpus de Jaboticabal. Foi utilizado um turbopulverizador com tanque com capacidade de $2.000 \mathrm{~L}$ de calda da marca KO Máquinas Agrícolas LTDA. - modelo mais utilizado na cultura de goiaba da região. $\mathrm{O}$ bocal de abastecimento do tanque é de $0,45 \mathrm{~m}$ de diâmetro e fica a $1,6 \mathrm{~m}$ de altura do solo. Foram utilizadas embalagens plásticas de PEAD de $1 \mathrm{~L}$, originais de agrotóxicos, com tampa plástica rígida de rosca e abertura de $4 \mathrm{~cm}$ de diâmetro. As embalagens vazias foram abastecidas com a calda aquosa, com $3,75 \mathrm{~g} / \mathrm{L}$ de sulfato de manganês ( $31 \%$ de $\mathrm{Mn}$ ). $\mathrm{O}$ cátion $\mathrm{Mn}$ foi utilizado como traçador (TÁCIO et al., 2008). Após o enchimento, os bocais foram lacrados com fita plástica adesiva para simular o lacre original da embalagem.

A atividade de preparo de caldas e abastecimento do tanque iniciou-se com a agitação vigorosa da embalagem, quebra do lacre, remoção da tampa, 
dosagem de $500 \mathrm{~mL}$ em proveta de vidro e deposição no tanque do turbopulverizador. Em seguida, colocação da tampa, agitação da embalagem fechada sem o lacre, nova dosagem de $500 \mathrm{~mL}$ e deposição no tanque. Em seguida, realização de tríplice lavagem da embalagem vazia. O procedimento da tríplice lavagem da embalagem foi iniciado com a colocação de $250 \mathrm{~mL}$ de água na embalagem vazia, fechamento com a tampa, agitação manual vigorosa por 30 segundos, retirada da tampa e deposição da água no tanque do turbopulverizador. Esse procedimento foi repetido por mais duas vezes.

As vestimentas avaliadas foram os conjuntos de proteção individual hidrorrepelentes; o modelo Agro Light, da empresa R\&B Equipamentos de Segurança, e o Kit Tratorizado, da AzeredoEPI. Os conjuntos hidrorrepelentes são compostos por camisa de mangas compridas, calça comprida, boné complementado com tecido protetor do pescoço e ombros, viseira de lâmina de acetato, afixada sobre a testa do trabalhador, e avental de tecido emborrachado na frente do corpo. Os dois conjuntos foram complementados com luvas de borracha nitrílica, botas de borracha e máscara descartável com filtro de carvão ativado. Cada condição de trabalho foi avaliada com dez repetições. As exposições médias avaliadas em cada uma das três condições de trabalho foram extrapoladas para uma jornada de trabalho com o abastecimento e 10 tanques/dia.

As exposições dérmicas foram avaliadas com macacões de brim branco com mangas compridas e capuz, para quantificar as exposições dérmicas da cabeça + pescoço, tronco (atrás e frente), braços e pernas (atrás e frente); luvas de algodão (mãos); absorventes higiênicos femininos (Carefree), afixados sobre máscaras descartáveis semifaciais (face) e sobre as botas de borracha (pés), de acordo com Machado Neto e Matuo (1989) e Machado Neto (1990). Após os períodos de exposições, as vestimentas amostradoras foram retiradas cuidadosamente do corpo do trabalhador, e os macacões, seccionados em partes com tesoura. As amostras das vestimentas foram acondicionadas separadamente em sacos plásticos flexíveis. O cátion Mn do sulfato de manganês coletado nas amostras das vestimentas foi solubilizado com uma solução extratora ácida $(\mathrm{HCl} 0,2 \mathrm{~N})$, adicionada diretamente nos sacos plásticos (OLIVEIRA , MACHADO NETO (2003b).

As exposições respiratórias foram avaliadas com bombas pessoais com fluxo de ar contínuo (2 L de ar/min), da marca A.P. Buck (OLIVEIRA; MACHADO NETO, 2003a). Foram utilizados cassetes de acrílico transparente com diâmetro interno de $37 \mathrm{~mm}$ e filtro de éster celulose, com porosidade de $0,8 \mu \mathrm{m}$. Após as exposições, os cassetes foram acondicionados em sacos plásticos flexíveis. A solução extratora do Mn foi colocada diretamente nos recipientes plásticos de $80 \mathrm{~mL}$ com os filtros e os suportes retirados dos cassetes. Em seguida, os frascos foram agitados manualmente e permaneceram em repouso por duas horas. A recuperação do cátion $\mathrm{Mn}$ no filtro de éster celulose foi previamente determinada em $92 \%$ (OLIVEIRA; MACHADO NETO, 2003a).

As exposições dérmicas e respiratórias às caldas foram calculadas com as quantidades do cátion Mn recuperadas de cada amostra e a concentração do Mn nas caldas contidas nas embalagens manuseadas.

As concentrações do Mn nas soluções extratoras foram determinadas por espectrofotometria de absorção atômica em chama de ar/acetileno oxidante, com lâmpada de cátodo oco, corrente de 5,0 mA, comprimento de onda de $279,5 \mathrm{~nm}$, fenda de $0,2 \mathrm{~nm}$. A curva-padrão de manganês foi obtida com solução de cloreto de manganês $(\mathrm{MnCl})$ Titrisol Merk 9988. A faixa de linearidade foi determinada com as concentrações de 0,$125 ; 0,25 ; 0,5 ; 1,0$ e 2,0 ppm $\left(\mathrm{R}^{2}=\right.$ 0,998). O limite de detecção foi de 0,019 ppm e o limite de quantificação, de 0,046 ppm, calculados de acordo com Skoog et al. (1998).

A eficiência (Efic.) dos conjuntos de vestimentas de proteção individual no controle das exposições dérmicas, em porcentagem, foi calculada com a fórmula:

Efic $=[(\mathbf{E D}+\mathbf{E D N C}) / \mathbf{E D}] \mathbf{x} 100($ TÁCIO et al. (2008), onde: $\mathrm{ED}=$ exposição Dérmica sem proteção e EDNC = Exposição dérmica Não Controlada pelas vestimentas de proteção.

A margem de segurança foi calculada com a fórmula: $\mathbf{M S}=($ NOEL $\times$ 70) $/($ QAE $\times 10)$ (SEVERN, 1984), onde: NOEL = Nível de Efeito Não Observável $(\mathrm{mg} / \mathrm{kg} /$ dia $)$, editado pela TGA (2008). $70=$ peso corpóreo médio do trabalhador. QAE $=$ Quantidade Absorvível da Exposição, que foi considerada como $10 \%$ na via dérmica (FELDMAN; MAIBACH, 1974; citados por BYERS et al., 1992) e $100 \%$ na respiratória. E $10=$ Fator de segurança (BROUWER et al., 1990).

As condições de trabalho foram classificadas em seguras ( $M S \geq 1)$ e inseguras ( $M S<1)$. Para as inseguras, foi calculada a necessidade de controle da exposição (NCE) com a fórmula: $\mathbf{N C E}=\left(\mathbf{1}-\mathbf{M S} \mathbf{S}_{<1}\right)$ x $100(\%)$, e o número de preparo de caldas seguro (NPCS), com a fórmula: NPCS $=$ MS x 10 tanques/ dia (MACHADO NETO, 2001),

As formulações líquidas dos agrotóxicos e suas recomendações, considerados neste trabalho, são as registradas para a cultura de goiaba (ANDREI, 2009; AGROFIT, 2009). 


\section{RESULTADOS E DISCUSSÃO}

$\mathrm{Na}$ atividade de preparo de caldas, no tanque do turbopulverizador de $2.000 \mathrm{~L}$, a quase totalidade da exposição de 13,354 mL de formulação/dia proporcionada ao trabalhador ocorre na via dérmica. Este resultado está de acordo com as exposições que ocorrem em diversas culturas agrícolas no Brasil (OLIVEIRA, 2000; OLIVEIRA; MACHADO NETO, 2003a) e no exterior (VAN HEMMEN, 1992). A exposição dérmica do preparador de caldas é superior à avaliada por Oliveira (2000). A maior exposição dérmica neste estudo é devida ao maior contato durante a realização da atividade. $\mathrm{O}$ trabalhador fez duas dosagens de $500 \mathrm{~mL}$ do conteúdo das embalagens ao abastecer o tanque, a abertura do lacre da tampa, simulado com a fita plástica adesiva, e a tríplice lavagem ao final da operação. No estudo de Oliveira (2000) não houve o fracionamento do conteúdo da embalagem nem a abertura do lacre da tampa.

Na distribuição da exposição na via dérmica no corpo do trabalhador, verifica-se que as mãos foram as partes mais expostas, com 70,63 \% da exposição dérmica potencial (Figura 1). Este resultado está de acordo com o de Oliveira (2000), para a atividade de preparo de calda.

A exposição dérmica não controlada pelas vestimentas com o conjunto de proteção individual Agro Light, da empresa R\&B, foi de $0,958 \mathrm{~mL}$ da formulação/dia e com o Kit Tratorizado, da AzeredoEPI, 0,773 mL de formulação/dia. Portanto, a eficiência destas vestimentas de proteção no controle da exposição do preparador de caldas foi de $92,8 \%$ para o conjunto Agro Light, e de 94,2 \% para o Kit Tratorizado, da AzeredoEPI.

Com o uso das vestimentas de proteção, a exposição dérmica não controlada foi mais distribuída nas partes do corpo do preparador de caldas, em relação à sem as vestimentas de proteção (Figura 1). Este resultado explica-se pela alta eficiência das luvas de borracha nitrílica na proteção das mãos ( $>97 \%$ ), que foram as partes mais expostas (Figura 1). Este resultado de eficiência das luvas de borracha está ligeiramente superior aos $95 \%$ de controle das exposições dérmicas das mãos, considerado como média nos estudos da área (LUNDEHN et al., 1992).

Com o uso do conjunto Agro Light, as partes mais expostas foram: pernas-atrás, com $20,82 \%$ do total, pernas-frente $(17,54 \%)$ e braços $(17,40 \%)$. Com o conjunto Kit Tratorizado, as partes mais expostas foram: pernas-atrás $(19,34$ $\%)$, pernas-frente $(18,25 \%)$ e braços $(16,89 \%)$.
Esses resultados estão de acordo com os relatados por Oliveira (2000), para o tratorista usando estes dois modelos de conjunto hidrorrepelente. Portanto, a distribuição da exposição dérmica não controlada pelas vestimentas de proteção individual está mais relacionada com a eficiência na proteção das mesmas do que com a atividade realizada. Este efeito é constatado nesta comparação de resultados entre a atividade de preparo de caldas e abastecimento do tanque e a de tratorista, ambas realizadas com o turbopulverizador.

A quantidade de calda que atinge a via respiratória do preparador de calda foi muito pequena (Tabela 2). Este resultado está de acordo com o de Tácio et al. (2008) para a atividade de tratorista, em aplicações de agrotóxicos em cultura de goiaba com o turbopul-verizador. A quantidade diminuta de agrotóxico contida neste volume de formulação não tem capacidade para intoxicar o trabalhador. Portanto, não há necessidade de qualquer medida de segurança para controlar esta exposição respiratória. Esta afirmativa não discorda ou contraria a legislação em vigor, a NR 31, ao determinar que as medidas de segurança devam ser adequadas aos riscos avaliados nas condições específicas de trabalhado com os agrotóxicos (BRASIL, 2005).

Como informação acessória, e para aproveitar a oportunidade do estudo, foi observada a ocorrência de embaçamento das viseiras durante a realização das atividades de preparo de calda. O embasamento das viseiras dos dois conjuntos de proteção individual foi rápido e permaneceu ao longo do período de trabalho (Figura 2). O embaçamento das viseiras ocorre porque estes EPIs ficam muito próximos da pele e concentram os vapores de água da transpiração da pele do rosto e da respiração do trabalhador. O boné com tecido protetor do pescoço e dos ombros também contribui com o embaçamento da viseira. Este EPI fecha as aberturas laterais da viseira e dificulta a movimentação do ar entre a região do rosto do trabalhador e o ambiente. O problema é que a viseira embaçada reduz a visibilidade do trabalhador e favorece a ocorrência de acidente de trabalho.

A classificação das três condições de trabalho pela segurança do trabalhador pode ser observada nos valores de MS e das necessidades de controle das exposições (Tabela 3). A condição de trabalho de preparo de caldas no tanque de $2.000 \mathrm{~L}$ do turbopulverizador, com as formulações liquidas de agrotóxicos registrados para a cultura de goiaba sem as vestimentas de proteção, classifica-se como insegura $(\mathrm{MS}<1)$. As necessidades de controle variam entre $73,8 \%$ e $100,0 \%$ para tornar estas condições de trabalho seguras, com MS pelo menos igual a 1. Estas 
condições de trabalho têm $100 \%$ de probabilidade de intoxicar os trabalhadores expostos devido às altas exposições que proporcionam, principalmente na via dérmica. As altas exposições dérmicas são devidas às altas concentrações dos ingredientes ativos nas formulações, entre 100 e $500 \mathrm{mg} / \mathrm{mL}$ da formulação, e os pequenos valores de NOEL, principalmente entre 0,02 e $2,0 \mathrm{mg} / \mathrm{kg} / \mathrm{dia}$ (Tabela 1 ).

Os conjuntos de equipamentos de proteção individual foram eficientes no controle das exposições às formulações proporcionadas pelas condições de trabalho ao preparador de caldas. A eficiência do conjunto Agro Light foi de 92,8\% das exposições, e do Kit Tratorizado, de 94,2 \%. Na atividade de tratorista em aplicação de agrotóxicos com o turbopulverizador em cultura de goiaba, a eficiência do conjunto Agro Light foi de 96,7 \%, e do Kit Tratorista, de 96,2 \% (TÁCIO et al., 2008). Verifica-se que os dois conjuntos de vestimentas de proteção são eficientes nas duas atividades diferentes existentes no trabalho de aplicação de agrotóxicos em cultura de citros com o turbopulverizador. Os dois conjuntos de vestimentas controlaram mais de $92 \%$ das exposições dérmicas e respiratórias proporcionadas ao tratorista e ao preparador de caldas.

Nas Tabelas 4 e 5, verifica-se que os controles das exposições em mais de $92 \%$ pelos dois conjuntos de vestimentas de proteção individual não foram suficientes para tornar as condições de trabalho de preparo de caldas seguras para nove dos dez agrotóxicos listados na Tabela 1. Apenas a condição de trabalho de preparo de caldas com o inseticida PROVADO (200 g de imidocloprido/L) classificase como segura com o uso dos dois conjuntos de vestimentas de proteção individual.

Embora as margens de segurança calculadas para o azoxistrobina, no AMISTAR TPO SC, e do trifloxistrobina, no NATIVO SC, são maiores que 1, as condições de trabalho de preparo de caldas com as duas formulações de misturas prontas classificamse como inseguras. A classificação da segurança às condições de trabalho com formulações com misturas prontas é realizada com base no menor valor da margem de segurança de um dos ingredientes ativos componentes da formulação.

Para os demais agrotóxicos considerados, há a necessidade de controle das exposições dérmicas não controladas pelas vestimentas de proteção, não obstante as altas eficiências dos dois conjuntos hidrorrepelentes avaliados. As necessidades de controle das exposições não controladas pelo conjunto de proteção individual denominado de Agro Light, da empresa R\&B, variaram entre 26,9 e $99,7 \%$ (Tabela 4 ), e entre 9,4 e $99,6 \%$ para o Kit
Tratorizado, da AzeredoEPI (Tabela 5). Portanto, estes conjuntos de vestimentas não foram suficientes para tornar estas condições de trabalho seguras, e outras medidas de segurança devem ser avaliadas, como as preventivas e de proteção coletiva, de acordo com a NR 31 (BRASIL, 2005). Por outro lado, o número de preparo de caldas seguro calculado para as três condições de trabalho (Tabelas 3; 4 e 5) variou, para as dez formulações líquidas de agrotóxicos registradas para a cultura de goiaba, de 0 a 0,5 tanques/dia para a atividade sem proteção; de 0 a 7 com o conjunto Agro Light, e de 0 a 9 com o Kit Tratorizado, que foi ligeiramente mais eficiente que o Agro Light. Esta atividade não deve ser realizada sem nenhuma medida de proteção, pois há risco de intoxicação do trabalhador mesmo no preparo de calda para apenas um tanque (Tabela 3). Entre as dez formulações, o LEBAYCID 500 CE destaca-se com o maior risco de intoxicação do preparador de caldas, com nenhum abastecimento seguro nas três condições de trabalho. $\mathrm{O}$ alto risco deste inseticida é devido à alta toxicidade do fenthion (Tabela 1), do grupo dos inseticidas organofosforados e inibidores das enzimas acetilcolinesterases (BURATTI et al., 2007). Porém, para as outras formulações, usar o número de preparo de caldas seguro destaca-se como uma medida de proteção coletiva adequada para ser associada aos conjuntos de proteção individual. Assim, cada trabalhador faz um determinado número de preparo de caldas seguro $(<10$ tanques/dia), de acordo com a formulação (Tabelas 4 e 5), e viabiliza a realização do trabalho em condições seguras, sob risco de intoxicação ocupacional aceitável. Assim, com o conjunto de vestimentas Agro Light, das formulações classificadas como inseguras (Tabela 4), o trabalhador pode trabalhar em condições seguras se preparar caldas para 5 ou 7 tanques/dia. Com o conjunto de vestimentas Kit Tratorizado, das formulações classificadas como inseguras (Tabela 5), o trabalhador pode trabalhar em condições seguras se preparar caldas para $6 ; 7$ ou 9 tanques/dia. 
TABELA 1 - Agrotóxicos registrados para a cultura da goiaba e respectivos valores de NOEL.

\begin{tabular}{|c|c|c|c|}
\hline Nome Comercial & Ingrediente Ativo & mg i.a./ mL p.c. & $\operatorname{NOEL}^{3}(\mathrm{mg} / \mathrm{kg} / \mathrm{dia})$ \\
\hline ALTO $100 \mathrm{CS}^{1 / 2}$ & ciproconazole & 100 & 1,00 \\
\hline \multirow{2}{*}{ AMISTAR TOP SC } & azoxistrobina + & 200 & 10,00 \\
\hline & difenoconazole & 125 & 1,00 \\
\hline CONDOR $200 \mathrm{SC}^{1 / 2}$ & bromuconazole & 200 & 2,00 \\
\hline COSTANT CE ${ }^{1 / 2}$ & tebuconazole & 200 & 1,50 \\
\hline ELITE CE $^{1 / 2}$ & tebuconazole & 200 & 1,50 \\
\hline FOLICUR 200 EC $^{2}$ & tebuconazole & 200 & 1,50 \\
\hline LEBAYCID $500 \mathrm{CE}^{1}$ & fenthion & 500 & 0,02 \\
\hline \multirow{2}{*}{ NATIVO SC ${ }^{2}$} & trifloxistrobina + & 100 & 5,00 \\
\hline & tebuconazole & 200 & 1,50 \\
\hline PROVADO $^{1}$ & imidacloprido & 200 & 6,00 \\
\hline TRÍADE CE CE $^{1 / 2}$ & tebuconazole & 200 & 1,50 \\
\hline
\end{tabular}

Fonte: Andrei (2009) ${ }^{1}$; AGROFIT (2009) $)^{2}$; TGA (2008) ${ }^{3}$

TABELA 2 - Exposições dérmica (ED) e respiratória (ER), totais (ED + ER) e não controladas pelas vestimentas de proteção individual proporcionadas ao preparador de caldas, no tanque de $2.000 \mathrm{~L}$ do turbopulverizador, utilizado na cultura de goiaba, e eficiência das vestimentas de proteção.

\begin{tabular}{|c|c|c|c|c|}
\hline \multirow[t]{2}{*}{ ATIVIDADES } & \multicolumn{3}{|c|}{$\begin{array}{c}\text { Exposições } \\
\mathrm{mL} \text { formulação/10 tanques/dia }\end{array}$} & \multirow{2}{*}{$\begin{array}{l}\text { Efic. } \\
(\%)\end{array}$} \\
\hline & ED & ER & (ED+ER) & \\
\hline Preparador de caldas (ET) & 13,3543 & $0,000153 *$ & 13,354453 & - \\
\hline Preparador de caldas com EPI Agro Light & 0,9581 & $0,000008^{*}$ & 0,958108 & 92,8 \\
\hline $\begin{array}{l}\text { Preparador de caldas com EPI Kit Tratorizado } \\
\text { Azeredo }\end{array}$ & 0,7726 & $0,000008^{*}$ & 0,772608 & 94,2 \\
\hline
\end{tabular}

$\mathrm{ED}=$ Exposição Dérmica; ER = Exposição Respiratória.

*ER não controlada pelos EPIs = estimada em 5 \% da ER (LUNDEHN et al. (1992).

TABELA 3 - Valores médios das exposições dérmicas e respiratórias totais (EDT) e (ERT), margens de segurança (MS), necessidade de controle da exposição (NCE) e número de preparo de caldas seguro na atividade de preparador de caldas, no tanque de $2.000 \mathrm{~L}$ do turbopulverizador utilizado na cultura da goiaba.

\begin{tabular}{|c|c|c|c|c|c|c|}
\hline \multirow[b]{2}{*}{ Nome Comercial } & \multirow[b]{2}{*}{ Ingrediente Ativo } & \multicolumn{2}{|c|}{ mg/dia } & \multirow[b]{2}{*}{ MS } & \multirow{2}{*}{$\begin{array}{c}\text { NCE } \\
(\%)\end{array}$} & \multirow{2}{*}{$\begin{array}{c}\text { Núm. de } \\
\text { Preparo } \\
\text { Seguro }\end{array}$} \\
\hline & & EDT & ERT & & & \\
\hline$\overline{\text { ALTO } 100 \mathrm{CS}^{1 / 2}}$ & ciproconazole & $1.335,43$ & 0,02 & 0,052 & 94,8 & 0,5 \\
\hline \multirow{2}{*}{ AMISTAR TOP SC ${ }^{1}$} & azoxistrobina + & $2.670,86$ & 0,03 & 0,262 & 73,8 & 2,6 \\
\hline & difenoconazole & $1.669,29$ & 0,02 & 0,042 & 95,8 & 0,4 \\
\hline CONDOR $200 \mathrm{SC}^{1 / 2}$ & bromuconazole & $2.670,86$ & 0,03 & 0,052 & 94,8 & 0,5 \\
\hline COSTANT CE ${ }^{1 / 2}$ & tebuconazole & $2.670,86$ & 0,03 & 0,039 & 96,1 & 0,4 \\
\hline ELITE CE $^{1 / 2}$ & tebuconazole & $2.670,86$ & 0,03 & 0,039 & 96,1 & 0,4 \\
\hline FOLICUR $200 \mathrm{EC}^{2}$ & tebuconazole & $2.670,86$ & 0,03 & 0,039 & 96,1 & 0,4 \\
\hline LEBAYCID $500 \mathrm{CE}^{1}$ & fenthion & $6.677,15$ & 0,08 & 0,000 & 100,0 & 0,0 \\
\hline \multirow{2}{*}{ NATIVO SC ${ }^{2}$} & trifloxistrobina + & $1.335,43$ & 0,02 & 0,262 & 73,8 & 2,6 \\
\hline & tebuconazole & $2.670,86$ & 0,03 & 0,039 & 96,1 & 0,4 \\
\hline PROVADO $^{1}$ & imidacloprido & $2.670,86$ & 0,03 & 0,157 & 84,3 & 1,6 \\
\hline TRÍADE $\mathrm{CE}^{1 / 2}$ & tebuconazole & $2.670,86$ & 0,03 & 0,039 & 96,1 & 0,4 \\
\hline
\end{tabular}

Fonte: Andrei (2009) ${ }^{1}$ e AGROFIT (2009)² 
TABELA 4 - Valores médios das exposições dérmicas não controladas (EDNC) e respiratórias não controladas (ERNC) pelas vestimentas de proteção com o conjunto Agro Light, exposições respiratórias (ER), margens de segurança (MS), necessidades de controle da exposição (NCE) e número de preparo de caldas seguro, na atividade de preparo de caldas, no tanque de 2.000 L do turbopulverizador utilizado na cultura da goiaba.

\begin{tabular}{|c|c|c|c|c|c|c|}
\hline \multirow{2}{*}{ Nome Comercial } & \multirow{2}{*}{ Ingrediente Ativo } & \multicolumn{2}{|c|}{$\mathrm{mg} / \mathrm{dia}$} & \multirow{2}{*}{ MS } & \multirow{2}{*}{$\begin{array}{l}\text { NCE } \\
(\%)\end{array}$} & \multirow{2}{*}{$\begin{array}{c}\text { Núm. de } \\
\text { Preparo } \\
\text { Seguro }\end{array}$} \\
\hline & & EDNC & ERNC & & & \\
\hline ALTO $100 \mathrm{CS}^{1 / 2}$ & ciproconazole & 95,81 & 0,00080 & 0,73 & 26,9 & 7,3 \\
\hline \multirow{2}{*}{ AMISTAR TOP SC ${ }^{1}$} & azoxistrobina + & 191,62 & 0,00160 & 3,65 & - & 36,5 \\
\hline & difenoconazole & 119,76 & 0,00100 & 0,58 & 41,6 & 5,8 \\
\hline CONDOR $200 \mathrm{SC}^{1 / 2}$ & bromuconazole & 191,62 & 0,00160 & 0,73 & 26,9 & 7,3 \\
\hline COSTANT CE ${ }^{1 / 2}$ & tebuconazole & 191,62 & 0,00160 & 0,55 & 45,2 & 5,5 \\
\hline ELITE CE $^{1 / 2}$ & tebuconazole & 191,62 & 0,00160 & 0,55 & 45,2 & 5,5 \\
\hline FOLICUR $200 \mathrm{EC}^{2}$ & tebuconazole & 191,62 & 0,00160 & 0,55 & 45,2 & 5,5 \\
\hline LEBAYCID $500 \mathrm{CE}^{1}$ & fenthion & 479,05 & 0,00400 & 0,00 & 99,7 & 0,0 \\
\hline \multirow{2}{*}{ NATIVO SC ${ }^{2}$} & trifloxistrobina + & 95,81 & 0,00080 & 3,65 & - & 36,5 \\
\hline & tebuconazole & 191,62 & 0,00160 & 0,55 & 45,2 & 5,5 \\
\hline PROVADO $^{1}$ & imidacloprido & 191,62 & 0,00160 & 2,19 & - & 21,9 \\
\hline TRÍADE CE ${ }^{1 / 2}$ & tebuconazole & 191,62 & 0,00160 & 0,55 & 45,2 & 5,5 \\
\hline
\end{tabular}

Fonte: Andrei (2009) ${ }^{1}$ e AGROFIT (2009) ${ }^{2}$

TABELA5 -Valores médios das exposições dérmicas não controladas (EDNC) e respiratórias não controladas (ERNC) pelas vestimentas com os conjuntos de proteção Kit Tratorizado, da Azeredo, exposições respiratórias totais (ERT), margens de segurança (MS), necessidade de controle da exposição (NCE) e número de preparo de caldas seguro, na atividade de preparador de caldas, no tanque de $2.000 \mathrm{~L}$ do turbopulverizador utilizado na cultura da goiaba.

\begin{tabular}{|c|c|c|c|c|c|c|}
\hline \multirow{2}{*}{ Nome Comercial } & \multirow{2}{*}{ Ingrediente Ativo } & \multicolumn{2}{|c|}{$\mathrm{mg} / \mathrm{dia}$} & \multirow{2}{*}{ MS } & \multirow{2}{*}{$\begin{array}{l}\text { NCE } \\
(\%)\end{array}$} & \multirow{2}{*}{$\begin{array}{c}\text { Núm. de } \\
\text { Pre p a r } \\
\text { Seguro }\end{array}$} \\
\hline & & EDNC & ERNC & & & \\
\hline ALTO $100 \mathrm{CS}^{1 / 2}$ & ciproconazole & 77,26 & 0,0008 & 0,91 & 9,4 & 9,1 \\
\hline \multirow{2}{*}{ AMISTAR TOP SC } & azoxistrobina + & 154,52 & 0,0016 & 4,53 & - & 45,3 \\
\hline & difenoconazole & 96,58 & 0,0010 & 0,72 & 27,5 & 7,2 \\
\hline CONDOR $200 \mathrm{SC}^{1 / 2}$ & bromuconazole & 154,52 & 0,0016 & 0,91 & 9,4 & 9,1 \\
\hline COSTANT CE ${ }^{1 / 2}$ & tebuconazole & 154,52 & 0,0016 & 0,68 & 32,0 & 6,8 \\
\hline ELITE CE ${ }^{1 / 2}$ & tebuconazole & 154,52 & 0,0016 & 0,68 & 32,0 & 6,8 \\
\hline FOLICUR $200 \mathrm{EC}^{2}$ & tebuconazole & 154,52 & 0,0016 & 0,68 & 32,0 & 6,8 \\
\hline LEBAYCID $500 \mathrm{CE}^{1}$ & fenthion & 386,30 & 0,0040 & 0,00 & 99,6 & 0,0 \\
\hline \multirow{2}{*}{ NATIVO SC ${ }^{2}$} & trifloxistrobina + & 77,26 & 0,0008 & 4,53 & - & 45,3 \\
\hline & tebuconazole & 154,52 & 0,0016 & 0,68 & 32,0 & 6,8 \\
\hline PROVADO $^{1}$ & imidacloprido & 154,52 & 0,0016 & 2,72 & - & 27,2 \\
\hline TRÍADE CE $\mathrm{CE}^{1 / 2}$ & tebuconazole & 154,52 & 0,0016 & 0,68 & 32,0 & 6,8 \\
\hline
\end{tabular}

Fonte: Andrei (2009) ${ }^{1}$ e AGROFIT (2009) $)^{2}$ 


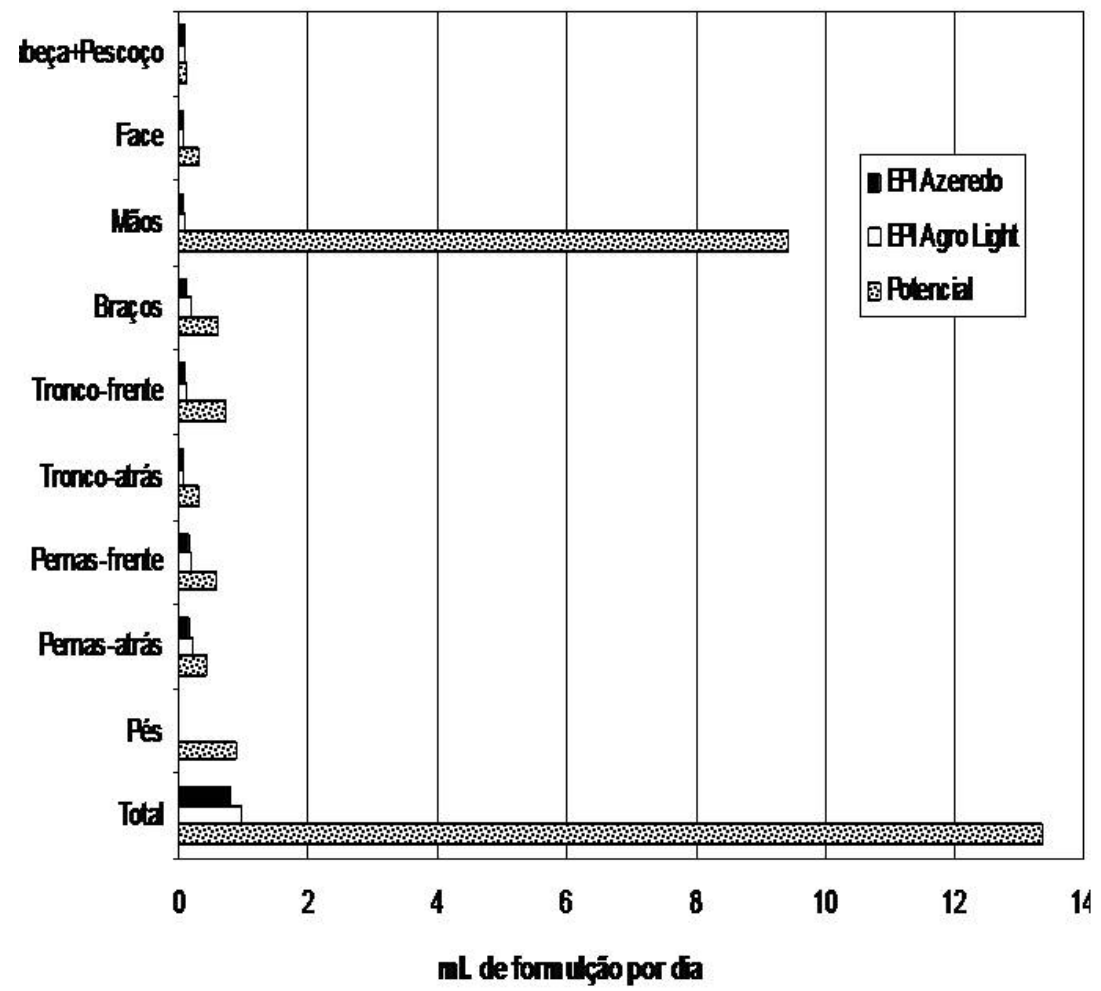

FIGURA 1 - Distribuição das exposições dérmicas nas diversas regiões do corpo do preparador de caldas, com e sem as vestimentas de proteção individual, no tanque de $2.000 \mathrm{~L}$ do turbopulverizador utilizado na cultura de goiaba.

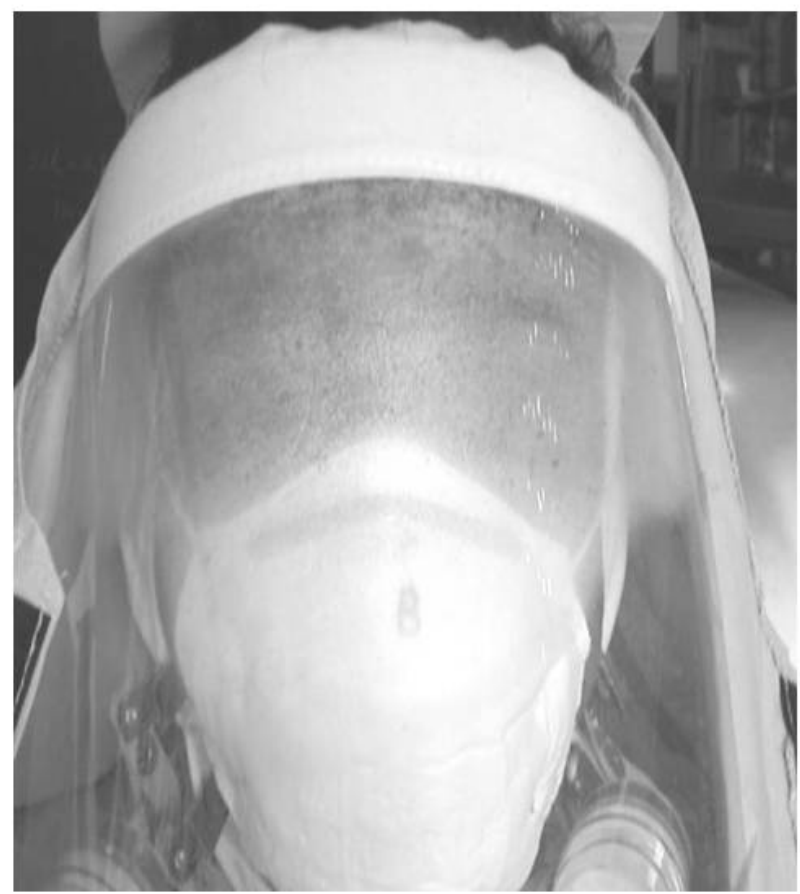

FIGURA 2 - Preparador de calda de agrotóxico com respirador semifacial descartável e viseira de lamina de acetato embaçada. 


\section{CONCLUSÕES}

Com os resultados obtidos nas condições de trabalho avaliadas, pode-se concluir que:

1- A exposição dérmica na atividade de preparo de caldas é de 13,35 mL de formulação/dia, e a respiratória, de $0,000153 \mathrm{~mL}$.

2- As mãos do trabalhador são as partes do corpo mais expostas, com $70,6 \%$ da exposição dérmica.

3- A eficiência do conjunto Agro Light, da empresa R\&B, é de $92,8 \%$ da exposição dérmica diária do preparador de caldas, e do Kit Tratorizado. da Azeredo, de 94,2 \%.

4- As exposições dérmicas não controladas pelos conjuntos de proteção individual distribuem-se nas diversas partes do corpo do trabalhador.

5- As atividades de preparo de caldas com as dez formulações líquidas de agrotóxicos registrados na cultura de goiaba são inseguras para o trabalhador.

6- Com as vestimentas de proteção individual, apenas a atividade de preparo de caldas com o inseticida PROVADO (200 g de imidacloprido/L) é segura.

7- Para as condições de trabalho inseguras, as necessidades de controle das exposições variam de 73,8 a 100\% (sem proteção), de 26,9 a 99,7\% (Agro Light) e de 9,4 a 99,6\% (Kit tratorizado).

8 - O número de preparos de calda seguros é de 0 tanques/dia sem proteção, de 0 a 5 ou 7 (Agro Light) ou de 6, 7 a 9 (Kit Tratorizado).

\section{AGRADECIMENTOS}

Os autores agradecem à Fundação de Amparo à Pesquisa do Estado de São Paulo - FAPESP, pelo auxílio financeiro concedido para a realização deste trabalho.

\section{REFERÊNCIAS}

ANDREI, E. Compêndio de defensivos agrícolas. 8.ed. São Paulo: Editora Andrei, 2009. CD-ROM.

AGROFIT. Sistema de agrotóxicos fitossanitários.

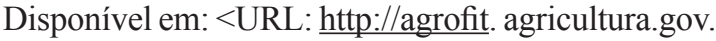
br/agrofit_cons/principal_agrofit_cons $>$. Acesso em: 09 dez. 2009.

BRASIL. Ministério do Trabalho. Norma regulamentadora de segurança e saúde no trabalho na agricultura, pecuária, silvicultura, exploração florestal e aquicultura - NR 31, Portaria n. 86 de 03 março 2005. Diário Oficial da União, 2005. 30p.

BROUWER, D. H.; BROUWER, R.; DE VREEDE, J.A.F.; DE MIK, G.; VAN HEMMEN, J.J. Respiratory exposure to field-strength dusts in greenhouses during application and after re-entry. The Hague: TNO Health Research, 1990. p.183-184. (Annual Report).

BURATTI, F. M.; LEONI C.; TESTAI, E. The human metabolism of organophosphoro-thionate pesticides: consequences for toxicological risk assessment. Journal für Verbraucherschutz und Lebensmittelsicherheit, , Basel: Birkhäuser, v.2, p.37- 44, 2007.

BYERS, M. E.; KAMBLE, S. T.; WITKOWSKI, J. F.; ECHTENKAMS, G. Exposure of a mixer-loader to insecticides applied to Corn via a center-pivot irrigation system. Bulletin of Environmental Contamination and Toxicology, New York, v.49, p.58-65, 1992.

LUNDEHN, J.; WESTPHAL, D.; KIECZKA, H.; KREBS, B.; LÖCHER-BOLZ, S.; MAASFELD, W.; PICK, E.D. Uniform principles for safeguarding the health of applicators of plant protection products: uniform principles for operator protection. Berlin: Kommissionsverlag Paul Parey, 1992. 90p.

MACHADO NETO, J. G. Determination of safe work time and exposure control need for pesticide applicators. Bulletin of Environmental Contamination and Toxicology, New York, v. 67, n. 1, p. 20-26, 2001. 
MACHADO NETO, J.G. Quantificação e controle da exposição dérmica de aplicadores de agrotóxicos na cultura estaqueada de tomate, na região de Cravinhos-SP. 1990. 112 f. Tese (Doutorado em Agronomia) - Faculdade de Ciências Agrárias e Veterinárias, Universidade Estadual Paulista, Jaboticabal, 1990.

OLIVEIRA, M.L. Segurança no trabalho de aplicação de agrotóxicos com turboatomizador e pulverizador de pistolas em citros, na região de Jaboticabal-SP. 2000. 99 f. Dissertação (Mestrado em Agronomia) - Faculdade de Ciências Agrárias e Veterinárias, Universidade Estadual Paulista, Jaboticabal.

OLIVEIRA, M. L.; MACHADO NETO, J. G. Use of Manganese as Tracer in the Determination of Respiratory Exposure and Relative Importance of Exposure Routes in the Safety of Pesticide Applicators in Citrus Orchards. Bulletin of Environmental Contamination and Toxicology, New York, v.70, p.415-421, 2003a.

OLIVEIRA, M.L.; MACHADO NETO, J.G. Uso do cátion manganês como traçador em avaliações da exposição dérmica aos agrotóxicos. Revista Brasileira de Toxicologia, Salvador, v.16, n.1, p.242, 2003 b. Suplemento
SEVERN, D.J. Use of exposure data for risk assessment. In: SIEWIERSKI, M. (Ed.). Determination and assessment of pesticide exposure. New York: Elsevier, 1984. p.13-19. (Studies in Environment Science, 24).

SKOOG, D. A.; HOLLER, F. J.; NIEMAN, T. A. Principles of instrumental analysis. $5^{\text {th }}$ ed. Austrália: Book/Cole, 1998. 849p.

TÁCIO, M. B.; OLIVEIRA, M. L. de; MACHADO NETO, J. G. Eficiência de vestimentas hidrorrepelentes novas na proteção do tratorista em pulverizações de agrotóxicos em goiaba com o turbopulverizador. Revista Brasileira de Fruticultura, Jaboticabal, v.30, n.1. p.106 - 111, 2008.

TGA. Therapeutic goods administration. Ad List: accept daily intakes for agricultural and veterinary chemicals. Australia: Australian Government, Department of Health and Ageing, Office of Chemical Safety, 2008. 98p.

VAN HEMMEN, J.J. Agricultural pesticide exposure data bases for risk assessment. Reviews of Environmental Reviews Contamination and Toxicology, New York, v.126, p.85, 1992. 